Research Article

\title{
Spatiotemporal Pattern in a Self- and Cross-Diffusive Predation Model with the Allee Effect
}

\author{
Feng Rao \\ College of Sciences, Nanjing University of Technology, Nanjing, Jiangsu 211816, China \\ Correspondence should be addressed to Feng Rao; raofeng2002@163.com
}

Received 16 April 2013; Accepted 12 June 2013

Academic Editor: Qiru Wang

Copyright (C) 2013 Feng Rao. This is an open access article distributed under the Creative Commons Attribution License, which permits unrestricted use, distribution, and reproduction in any medium, provided the original work is properly cited.

\begin{abstract}
This paper proposes and analyzes a mathematical model for a predator-prey interaction with the Allee effect on prey species and with self- and cross-diffusion. The effect of diffusion which can drive the model with zero-flux boundary conditions to Turing instability is investigated. We present numerical evidence of time evolution of patterns controlled by self- and cross-diffusion in the model and find that the model dynamics exhibits a cross-diffusion controlled formation growth to spotted and striped-like coexisting and spotted pattern replication. Moreover, we discuss the effect of cross-diffusivity on the stability of the nontrivial equilibrium of the model, which depends upon the magnitudes of the self- and cross-diffusion coefficients. The obtained results show that cross-diffusion plays an important role in the pattern formation of the predator-prey model. It is also useful to apply the reaction-diffusion model to reveal the spatial predation in the real world.
\end{abstract}

\section{Introduction}

It is well known that predator-prey interaction is one of the basic interspecies relations for ecological and social models. The mathematical model of predator-prey type has played a major role in the studies of biological invasion of foreign species, epidemics spreading, and extinction/spread of flame balls in combustion or autocatalytic chemical reaction [1]. The first differential equation model of predator-prey type Lotka-Volterra equation was formulated by Lotka and Volterra in 1920s, when attempts were first made to find ecological laws of nature $[2,3]$. In recent years, one of the important predator-prey models is the Holling type II model, originally due to Holling $[4,5]$, which has been studied in many articles; see, for example, [6-12].

In population dynamics, any mechanism that can lead to the positive relationship between population density and the per capita growth rate can be termed an Allee effect [13-15], starting with the pioneer work of Allee [16]. The Allee effect can be caused by a number of sources such as difficulties in finding mates at small densities, reproductive facilitation, predation, environment conditioning, and inbreeding depression. This effect usually saturates or disappears as populations get larger $[17,18]$. The Allee effect's strong potential impact on population dynamics has been attracting much attention recently. Detailed investigations relating to the Allee effect may be found in [19-33].

On the other hand, in nature, there is a tendency that the preys would keep away from predators and the escape velocity of the preys may be taken as proportional to the dispersive velocity of the predators. In the same manner, there is a tendency that the predators would get closer to the preys and the chase velocity of predators may be considered to be proportional to the dispersive velocity of the preys $[34,35]$. In view of these, cross-diffusion arises, which was proposed first by Kerner [36] and first applied in competitive population system by Shigesada et al. [37] to describe the population pressure by other species. Cross-diffusion expresses the population fluxes of one species due to the presence of the other species. In the realistic situation, self-diffusion implies passive diffusion where the diffusing species moves along its concentration gradient, while cross-diffusion implies countertransport. The cross-diffusion coefficient may be positive or negative. When it is positive, one species moves along the concentration gradient of another species, and when it is negative, one species moves against the concentration gradient of another species $[38,39]$. 
Among theoretical and mathematical biologists, there has been considerable interest to investigate the dynamical behavior of a predation system of interacting populations by taking into account the effect of self- and cross-diffusion [40-48]. But in the studies on spatiotemporal dynamics of a Holling type II predator-prey model with an Allee effect on prey, little attention has been paid to study the effect of selfand cross-diffusion. Based on the previous discussions, the main purpose of this paper is to examine out the stability behavior of the Holling-type-II predator-prey model with Allee effect on prey by taking into account self- and crossdiffusion.

The organization of this paper is as follows. In Section 2, we present a Holling-type-II predation model in the presence of Allee effect with self- and cross-diffusion. In Section 3, we analyze the model and derive the condition of Turing instability with respect to parameters. Furthermore, we use numerical simulations to reveal the emergence of different patterns and the influence of cross-diffusion on the dynamical behavior of the model. Finally, a brief discussion is given in Section 4.

\section{Model}

In this paper, we consider the following model of twodimensional (i.e., $2 D$ ) population dynamics:

$$
\begin{aligned}
\dot{N} & =F(N)-f(N, P), \\
\dot{P} & =\mu f(N, P)-M P,
\end{aligned}
$$

where $N$ and $P$ are the densities of prey and predator at time $T>0$, respectively. The function $F(N)$ describes prey multiplication, $f(N, P)$ describes predation, and the term $M P$ stands for predator mortality. $\mu$ is the food utilization coefficient. We assume that the predator response is of Holling-type-II which is usually parameterized as follows:

$$
f(N, P)=A \frac{N P}{N+B},
$$

where $A$ is the predation rate and $B$ is the half-saturation density.

Referring to [18], we assume that Allee dynamics for the prey population, its growth rate can be parameterized as follows:

$$
F(N)=\frac{4 \omega}{\left(K-N_{0}\right)^{2}} N\left(N-N_{0}\right)(K-N),
$$

where $K$ is the prey carrying capacity, $\omega$ is the maximum per capita growth rate, and $N_{0}$ is the "threshold" $\left(N_{0}<K\right)$, so that for $N<N_{0}$, the growth rate becomes negative. The value of $N_{0}$ can be considered as a measure of the intensity of the Allee effect: the less the value of $N_{0}$ is, the less prominent is the Allee effect [21]. In other words, the Allee effect is called "strong" if $0<N_{0}<K$ (when the growth rate becomes negative for $N<N_{0}$ ) and "weak" if $-K<N_{0} \leq 0$. For $N_{0} \leq-K$, the Allee effect is absent [49].
Based on the Holling-type-II predator-prey model with the Allee effect for the prey, in the following, we investigate the dynamical complexity induced by self- and crossdiffusion in the model. By choosing appropriate scales for the variables of (1), (2), and (3), the number of parameters can be lessened. Considering dimensionless variables as in [21] with the following scaling:

$$
u=\frac{N}{K}, \quad v=\frac{P}{\mu K}, \quad t=\frac{\mu A K T}{B},
$$

we obtain and employ the corresponding diffusive model as follows:

$$
\begin{gathered}
\frac{\partial u}{\partial t}=\gamma u(u-\beta)(1-u)-\frac{u v}{1+\alpha u}+D_{11} \nabla^{2} u+D_{12} \nabla^{2} v \\
\frac{\partial v}{\partial t}=\frac{u v}{1+\alpha u}-\delta v+D_{21} \nabla^{2} u+D_{22} \nabla^{2} v
\end{gathered}
$$

Here,

$$
\begin{gathered}
\alpha=\frac{K}{B}, \quad \beta=\frac{N_{0}}{K}, \\
\gamma=\frac{4 \omega B K}{\mu A\left(K-N_{0}\right)^{2}}, \quad \delta=\frac{M B}{\mu A K} .
\end{gathered}
$$

In model (5), the nonnegative constants $D_{11}$ and $D_{22}$ are the self-diffusion coefficients of $u$ and $v$, respectively, which imply the movement of individuals from a higher to lower concentration region. The cross-diffusion coefficients $D_{12}$ and $D_{21}$ express the respective population fluxes of the preys and predators resulting from the presence of the other species, respectively. That is to say, $D_{12}$ in (5) represents the tendency of the prey $u$ to keep away from its predators $v$, and $D_{21}$ represents the tendency of the predator to chase its prey. The cross-diffusion coefficients, $D_{12}$ and $D_{21}$, may be positive or negative. Positive cross-diffusion coefficient denotes that one species tends to move in the direction of lower concentration of another species, and negative crossdiffusion expresses the population fluxes of one species in the direction of higher concentration of another species $[39,50]$. Note that $\nabla^{2}=\partial^{2} / \partial x^{2}+\partial^{2} / \partial y^{2}$ is the usual Laplacian operator in a two-dimensional space.

Model (5) is to be analyzed with the following nonzero initial conditions:

$$
\begin{gathered}
u(x, y, t)>0, \quad v(x, y, t)>0, \\
(x, y) \in \Omega=[0, L x] \times[0, L y]
\end{gathered}
$$

and the following zero-flux boundary conditions:

$$
\frac{\partial u}{\partial \mathbf{n}}=\frac{\partial v}{\partial \mathbf{n}}=0, \quad(x, y) \in \partial \Omega .
$$

In the previous part, $L x$ and $L y$ give the size of the model in the directions of $x$ and $y$, respectively. $\mathbf{n}$ is the outward unit normal vector of the boundary $\partial \Omega$ which we will assume to be smooth. Zero-flux boundary conditions imply that no external input is imposed from outside $[1,51]$. 


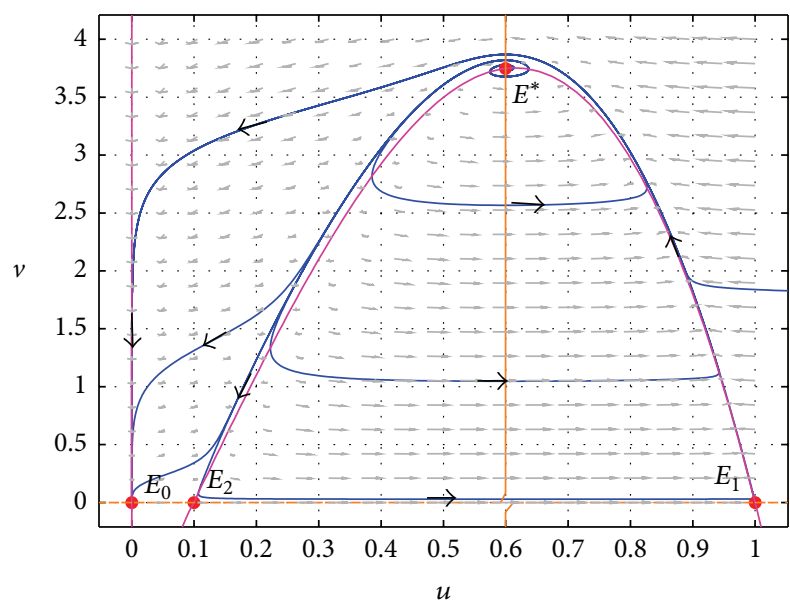

FIgURE 1: Phase portrait of model (5). The parameters are taken as $\alpha=1.11, \beta=0.1, \gamma=11.25$, and $\delta=0.36$. The horizontal axis is the prey population $u$, and the vertical axis is the predator population $v$. $E_{0}=(0,0)$ is a stable node, $E_{1}=(1,0)$ and $E_{2}=(0.1,0)$ are saddle points, and $E^{*}=(0.6,3.75)$ is unstable.

For model (5), without diffusion terms, there are four stationary states: $E_{0}=(0,0), E_{1}=(1,0), E_{2}=(\beta, 0)$, and the coexistence state $E^{*}=\left(u^{*}, v^{*}\right)$, where

$$
u^{*}=\frac{\delta}{1-\alpha \delta}, \quad v^{*}=\gamma\left(u^{*}-\beta\right)\left(1-u^{*}\right)\left(1+\alpha u^{*}\right) .
$$

Using the standard linear stability analysis, it can be readily seen that

(i) $E_{0}=(0,0)$ is always a stable node and corresponds to extinction of both prey and predator;

(ii) two uniform steady states $E_{1}=(1,0)$ and $E_{2}=(\beta, 0)$ are either nodes or saddle points, depending on the relation between the parameters, and correspond to extinction of the predator;

(iii) the coexistence state $E^{*}=\left(u^{*}, v^{*}\right)$ lies in the biologically meaningful domain $u>0, v>0$ under condition $\beta<u^{*}<1$ (for these parameter values $E_{1}=(1,0)$ and $E_{2}=(\beta, 0)$ are saddle points $)$ and it can be either a stable or unstable focus or a node.

For more details about model (5) without diffusions, we can refer to reference [29].

When we take the parameters as $\alpha=1.11, \beta=0.1$, $\gamma=11.25$, and $\delta=0.36$ for $(5), E_{0}=(0,0)$ is a stable node, $E_{1}=(1,0)$ and $E_{2}=(0.1,0)$ are saddle points, and the positive equilibrium $E^{*}=(0.6,3.75)$ exists, which is unstable and shown in Figure 1.

\section{Pattern Formation}

To study patterns of the self- and cross-diffusion model given by (5), we must consider a spatially homogeneous system.

In this section, we perform extensive numerical simulations of the spatially extended model (5) in a two-dimensional space, and the qualitative results are shown here. All our numerical simulations employ the zero-flux boundary conditions (8) with a system size of $L x \times L y$ discretized through $x \rightarrow\left(x_{0}, x_{1}, x_{2}, \ldots, x_{n}\right)$ and $y \rightarrow\left(y_{0}, y_{1}, y_{2}, \ldots, y_{n}\right)$ with $n=200$, and the time step of $t=1 / 100$.

We use the standard five-point approximation for the $2 D$ Laplacian with the zero-flux boundary conditions $[52,53]$. That is, the concentrations $\left(u_{i, j}^{n+1}, v_{i, j}^{n+1}\right)$ at the time $(n+1) t$ at the mesh position $\left(x_{i}, y_{j}\right)$ are given by

$$
\begin{aligned}
& u_{i, j}^{n+1}=u_{i, j}^{n}+t D_{11} \Delta_{h} u_{i, j}^{n}+t D_{12} \Delta_{h} v_{i, j}^{n}+t f\left(u_{i, j}^{n}, v_{i, j}^{n}\right), \\
& v_{i, j}^{n+1}=v_{i, j}^{n}+t D_{21} \Delta_{h} u_{i, j}^{n}+t D_{22} \Delta_{h} v_{i, j}^{n}+t g\left(u_{i, j}^{n}, v_{i, j}^{n}\right),
\end{aligned}
$$

with the Laplacian defined by

$$
\Delta_{h} u_{i, j}^{n}=\frac{u_{i+1, j}^{n}+u_{i-1, j}^{n}+u_{i, j+1}^{n}+u_{i, j-1}^{n}-4 u_{i, j}^{n}}{h^{2}},
$$

where the space stepsize $h=1 / 3$.

We are mainly interested in investigating the behavior of model (5) around the interior equilibrium point; so we will put emphasis on the positive equilibrium point $E^{*}=$ $\left(u^{*}, v^{*}\right)$. The entire system is initially placed in the stationary state $\left(u^{*}, v^{*}\right)$, and the propagation velocity of the initial perturbation is thus of the order of $5 \times 10^{-4}$ space units per time unit. After the initial period during which the perturbation spreads, either the system goes into a timedependent state or goes into a time-independent steady state [54].

To study the effect of diffusion on model (5), we consider the linearized form of the model about $E^{*}=\left(u^{*}, v^{*}\right)$ as follows:

$$
\begin{aligned}
& \frac{\partial \widehat{u}}{\partial t}=J_{11} \widehat{u}+J_{12} \widehat{v}+D_{11} \nabla^{2} \widehat{u}+D_{12} \nabla^{2} \widehat{v}, \\
& \frac{\partial \widehat{v}}{\partial t}=J_{21} \widehat{u}+J_{22} \widehat{v}+D_{21} \nabla^{2} \widehat{u}+D_{22} \nabla^{2} \widehat{v},
\end{aligned}
$$

where $u=u^{*}+\widehat{u}, v=v^{*}+\widehat{v}$ and

$$
\begin{gathered}
J_{11}=-\left(d \left((b-a-a b) n^{2}+a^{2} d(2 n+d)\right.\right. \\
\left.\left.+(a b+a+b) d^{2}-2 b d n\right)\right) \\
\times\left(b n(n-d)^{2}\right)^{-1}, \\
J_{12}=-\frac{d m}{n}, \quad J_{21}=-\frac{(a d+d-n)(a d+b d-b n)}{b m(n-d)}, \\
J_{22}=0 .
\end{gathered}
$$

We note that $(\widehat{u}, \widehat{v})$ are small perturbations of $(u, v)$ about the equilibrium point $\left(u^{*}, v^{*}\right)$ and the stationary state can become unstable to a nonuniform perturbation

$$
\begin{gathered}
\widehat{u}(\mathbf{r}, t) \sim \exp (\lambda t+\mathrm{i} \vec{k} \cdot \mathbf{r}), \\
\widehat{v}(\mathbf{r}, t) \sim \exp (\lambda t+\mathrm{i} \vec{k} \cdot \mathbf{r}), \quad \mathbf{r}=(x, y) .
\end{gathered}
$$


Any solution of model (12) can be expanded into a Fourier series so that

$$
\begin{aligned}
& \widehat{u}(\mathbf{r}, t)=\sum_{i, j=0}^{\infty} u_{i j}(\mathbf{r}, t)=\sum_{i, j=0}^{\infty} \alpha_{i j}(t) \sin (\vec{k} \mathbf{r}), \\
& \widehat{v}(\mathbf{r}, t)=\sum_{i, j=0}^{\infty} v_{i j}(\mathbf{r}, t)=\sum_{i, j=0}^{\infty} \beta_{i j}(t) \sin (\vec{k} \mathbf{r}),
\end{aligned}
$$

where $0<x<L x, 0<y<L y$. Note that $\vec{k}=\left(k_{i}, k_{j}\right), k_{i}=$ $i \pi / L x$, and $k_{j}=j \pi / L y$ are the corresponding wavenumbers.

Substituting $u_{i j}$ and $v_{i j}$ into (12), we obtain

$$
\begin{gathered}
\frac{\mathrm{d} \alpha_{i j}}{\mathrm{~d} t}=\left(J_{11}-D_{11} k^{2}\right) \alpha_{i j}+\left(J_{12}-D_{12} k^{2}\right) \beta_{i j}, \\
\frac{\mathrm{d} \beta_{i j}}{\mathrm{~d} t}=\left(J_{21}-D_{21} k^{2}\right) \alpha_{i j}-D_{22} k^{2} \beta_{i j},
\end{gathered}
$$

where $k^{2}=k_{i}^{2}+k_{j}^{2}$. The characteristic equation of the linearized system is given by

$$
\lambda^{2}-\operatorname{tr}(\mathscr{J}) \lambda+\operatorname{det}(\mathscr{J})=0
$$

where

$$
\begin{gathered}
\mathscr{J}=\left(\begin{array}{cc}
J_{11}-D_{11} k^{2} & J_{12}-D_{12} k^{2} \\
J_{21}-D_{21} k^{2} & -D_{22} k^{2}
\end{array}\right), \\
\operatorname{tr}(\mathscr{J})=J_{11}-\left(D_{11}+D_{22}\right) k^{2}, \\
\operatorname{det}(\mathscr{J})=\operatorname{det}(D) k^{4}+\left(D_{12} J_{21}+D_{21} J_{12}-D_{22} J_{11}\right) k^{2} \\
-J_{12} J_{21} .
\end{gathered}
$$

Here, the diffusive matrix is

$$
D=\left(\begin{array}{ll}
D_{11} & D_{12} \\
D_{21} & D_{22}
\end{array}\right)
$$

and the determinant of $D$ is $\operatorname{det}(D)=D_{11} D_{22}-D_{12} D_{21}$. Then the roots of (17) yield the dispersion relation

$$
\lambda_{1,2}=\frac{1}{2}\left(\operatorname{tr}(\mathscr{J}) \pm \sqrt{(\operatorname{tr}(\mathscr{J}))^{2}-4 \operatorname{det}(\mathscr{J})}\right) .
$$

In order to have diffusion-induced instability (i.e., Turing instability), which means that it is an asymptotically stable equilibrium of the diffusion-less model (5) but is unstable with respect to solutions of the spatial model (5), at least one of the conditions $\operatorname{tr}(\mathscr{F})<0$ and $\operatorname{det}(\mathscr{J})>0$ must be violated by Routh-Hurwitz criterion. It is evident that the first condition $\operatorname{tr}(\mathscr{J})<0$ is not violated when the condition $J_{11}<0$ is met. Hence, in order to obtain diffusion instability, we need $\operatorname{det}(\mathscr{J})<0$. Thus, the condition is given by

$$
\begin{aligned}
G\left(k^{2}\right)= & \operatorname{det}(D) k^{4}+\left(D_{12} J_{21}+D_{21} J_{12}-D_{22} J_{11}\right) k^{2} \\
& -J_{12} J_{21}<0 .
\end{aligned}
$$

If the minimum of $G\left(k^{2}\right)$ is negative, then a range of modes $k_{-}^{2}<k^{2}<k_{+}^{2}$ will grow, where

$$
\begin{aligned}
k_{-}^{2}= & \frac{1}{2 \operatorname{det}(D)} \\
& \times\left[D_{22} J_{11}-D_{21} J_{12}-D_{12} J_{21}\right. \\
& \quad-\left(D_{22}^{2} J_{11}^{2}+\left(D_{21} J_{12}+D_{12} J_{21}\right)^{2}+4 J_{12} J_{21} \operatorname{det}(D)\right. \\
k_{+}^{2}=\frac{1}{2 \operatorname{det}(D)} & \left.\left.-2 D_{22} J_{11}\left(D_{21} J_{12}+D_{12} J_{21}\right)\right)^{1 / 2}\right], \\
& \times\left[D_{22} J_{11}-D_{21} J_{12}-D_{12} J_{21}\right. \\
& \quad+\left(D_{22}^{2} J_{11}^{2}+\left(D_{21} J_{12}+D_{12} J_{21}\right)^{2}+4 J_{12} J_{21} \operatorname{det}(D)\right. \\
& \left.\left.-2 D_{22} J_{11}\left(D_{21} J_{12}+D_{12} J_{21}\right)\right)^{1 / 2}\right] .
\end{aligned}
$$

In addition, when

$$
k^{2}=k_{c}^{2}=\frac{D_{22} J_{11}-D_{12} J_{21}-D_{21} J_{12}}{2 \operatorname{det}(D)}>0,
$$

we obtain

$$
G_{\text {min }}=-J_{12} J_{21}-\frac{\left(D_{22} J_{11}-D_{12} J_{21}-D_{21} J_{12}\right)^{2}}{4 \operatorname{det}(D)} .
$$

Then we have

$$
G\left(k^{2}\right)<0 \Longleftrightarrow k_{-}^{2}<k^{2}<k_{+}^{2} .
$$

If both $k_{-}^{2}$ and $k_{+}^{2}$ exist and have positive values, they limit the range of instability for a locally stable equilibrium (see Figure 2).

Summarizing the above discussions, we can obtain the following theorem.

Theorem 1. The criterion for Turing instability for model (5) satisfies the following condition:

$$
D_{22} J_{11}-D_{12} J_{21}-D_{21} J_{12}>2 \sqrt{-J_{12} J_{21} \operatorname{det}(D)}>0 \text {. }
$$

In Figure 3, we plot the dispersion relation corresponding to several values of parameter $D_{12}$ while keeping the others fixed as

$$
\begin{gathered}
\alpha=1.11, \quad \beta=0.1, \\
\gamma=11.25, \quad \delta=0.36, \\
D_{11}=0.2, \quad D_{22}=2, \quad D_{21}=-0.4 .
\end{gathered}
$$




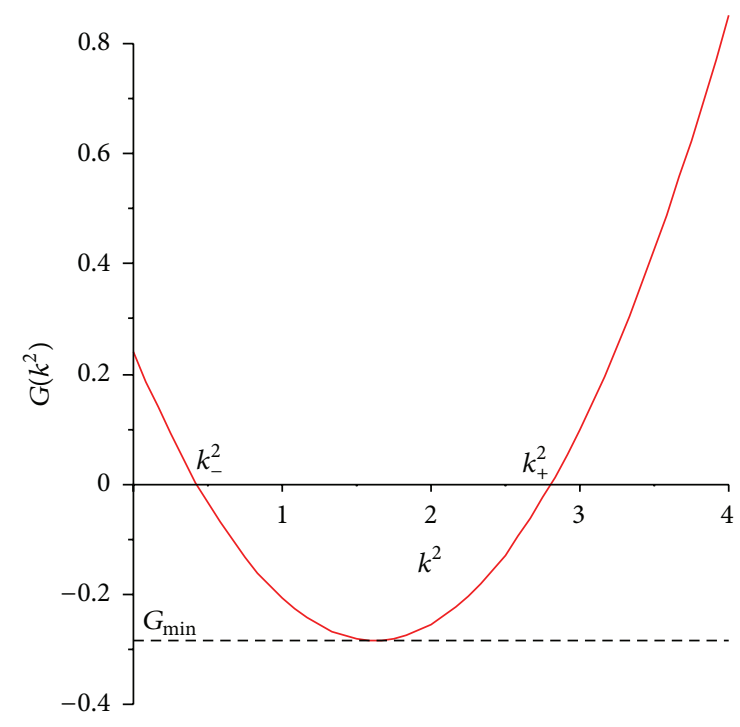

FIGURE 2: Model (5) with wavenumbers lying between the zeros of $G\left(k^{2}\right), k_{-}^{2}$, and $k_{+}^{2}$ growing in the Turing instability. Parameters are taken as $\alpha=1.11, \beta=0.1, \gamma=11.25, \delta=0.36, D_{11}=0.2, D_{22}=$ 2, $D_{12}=-0.5$, and $D_{21}=-0.4$; hence, $k_{-}^{2}=0.427433403, k_{+}^{2}=$ 2.807586678 , and $G_{\min }=-0.283261256$.

Curve (B) in Figure 3 corresponds to the critical Turing value $D_{12}^{\mathrm{T}}=-0.31$. When the parameter $D_{12}=-1.6$ below the $D_{12}^{\mathrm{T}}$ (i.e., curve (A) in Figure 3 ), the Turing instability occurs, while $D_{12}=0.4$ above the $D_{12}^{\mathrm{T}}$ (i.e., curve (C) in Figure 3), the Turing instability decays. That is to say, when $D_{12}>D_{12}^{\mathrm{T}}$, the steady state is the only stable solution of model (5). The spatial patterns are generated when $D_{12}$ passes through the critical Turing bifurcation point $D_{12}^{\mathrm{T}}$.

Figure 4 shows the spatial patterns of prey $u$ and predator $v$ and the corresponding phase portraits of model (5). Figures 4(a1) and 4(a2) show stationary spotted and striped-like patterns emerge mixed in the distribution of prey $u$ and predator $v$ population density, respectively. In Figures $4(\mathrm{al})$ and $4(\mathrm{a} 2)$, when $D_{12}=-0.365<D_{12}^{\mathrm{T}}=-0.31$, starting with a homogeneous state $(0.6,3.75)$, the random perturbations make the formations end with spotted and striped-like coexistence in the spatial domain at $t=10000$ (i.e., 1000000 iterations), and the mixed patterns prevail over the whole domain at last. In Figure 4(a3), one can see that as $t \rightarrow \infty, u$ and $v$ coordinates fix to $(0.745,3.428)$ which is the steady state.

Figures 4(b1) and 4(b2) show spotted pattern formations of prey $u$ and predator $v$, respectively. In Figures $4(\mathrm{bl})$ and $4(\mathrm{~b} 2), D_{12}=-0.5<D_{12}^{\mathrm{T}}=-0.31$, starting with a homogeneous state $(0.6,3.75)$, the random perturbations make the formations end with a time-independent spottedpatterns at $t=10000$ (i.e., 1000000 iterations). From Figures 4 (b1) and 4(b2), we can see that the spotted patterns prevail over the whole domain and the dynamics of model (5) do not undergo any further changes. In Figure 4(b3), which is a phase portrait, the steady state $(0.725,3.061)$ exists and is asymptotically stable. From the figure, we can see that $(u, v) \rightarrow(0.725,3.061)$ with time.

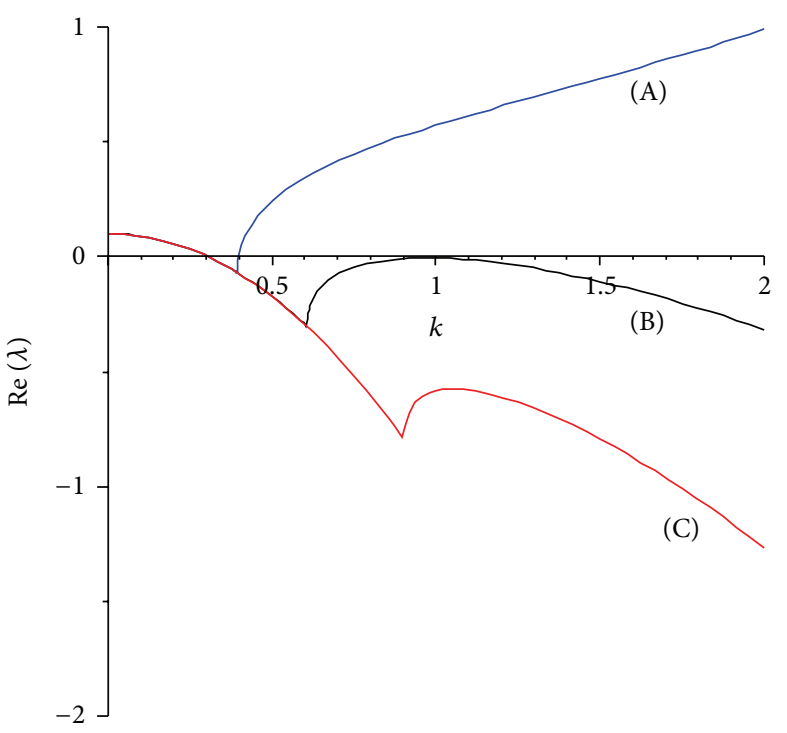

FIGURE 3: Variation of dispersion relation of model (5) for different $D_{12}$ : (A) $D_{12}=-1.6<D_{12}^{\mathrm{T}}$, the Turing instability occurs; (B) $D_{12}=$ $D_{12}^{\mathrm{T}}=-0.31$, the critical Turing value; and $(\mathrm{C}) D_{12}=0.4>D_{12}^{\mathrm{T}}$, the Turing instability decays. Other parameters values are fixed as (27) in the text.

In Figure 4, two different snapshots during the temporal evolution of model (5) are presented in a two-dimensional space under different $D_{12}$ values. These results indicate that the effect of cross-diffusion $D_{12}$ for pattern formation is tremendous, and model (5) is conditionally stable at unique positive equilibrium.

In order to investigate quantitatively the evolution of model (5), we depict the typical emergence of Turing pattern in the spacetime plots of Figure 5. The method of spacetime plots is to let $y$ be a constant, choose the line $y=100$ from each pattern snapshot, and pile these lines in time order [5559]. Figures 5(a) and 5(b) show the evolution process of prey $u$ throughout time $t$ and space $x$ with different $D_{12}$, respectively, where time increases from bottom to top, and the horizontal axis represents the spatial location. From Figures 5(a) and $5(\mathrm{~b})$, the single parameter of model (5) namely $D_{12}$, which is the diffusion coefficient of the prey, can lead to dramatic changes in the qualitative dynamics of solutions. From a biological perspective, the cross term plays a constructive role in the pattern formation of the prey, that is, changes the Turing pattern into regular spatial pattern with time.

According to the works of many researchers [53, 60-62], the choice of initial conditions can affect the spatiotemporal dynamics of a reaction-diffusion model in ecosystems. In Figure 6, when $D_{12}=-0.5$ and other parameters are fixed as (27), we choose the special initial condition of model (5) to investigate the evolution of the spatial pattern of prey $u$ at a different evolution time. The initial condition is introduced as the following form:

$$
u(x, y, 0)=u^{*}, \quad v(x, y, 0)=v^{*}, \quad 90<x, y<110,
$$

which is a square. Initially, the entire system is placed in the stationary state $\left(u^{*}, v^{*}\right)=(0.6,3.75)$, and the propagation 


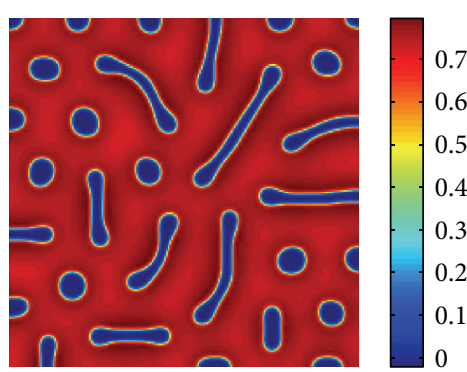

(a1)

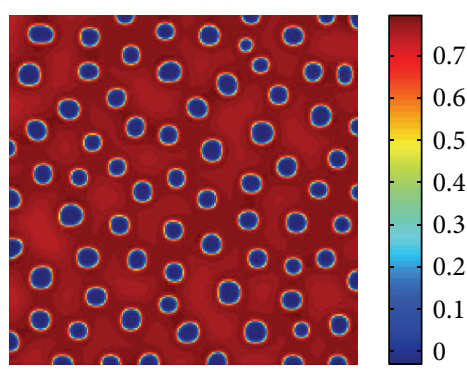

(b1)

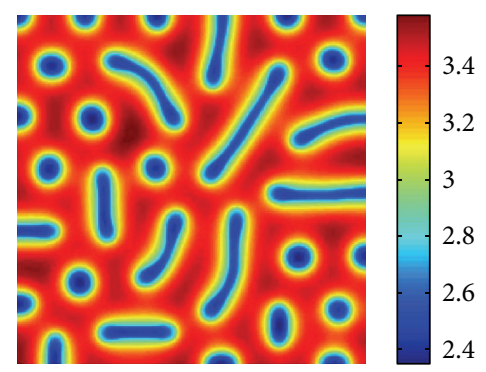

(a2)

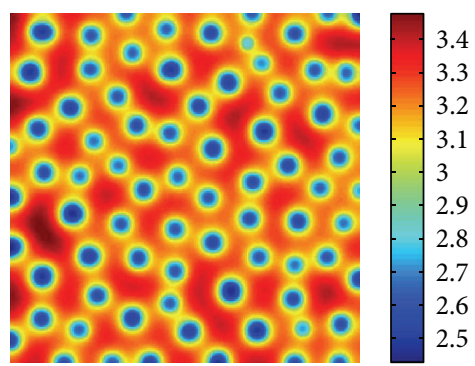

(b2)

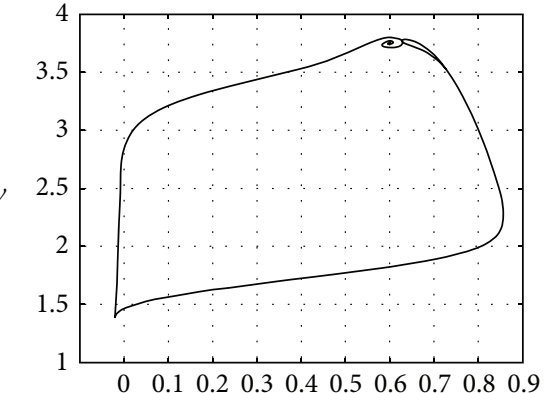

(a3)

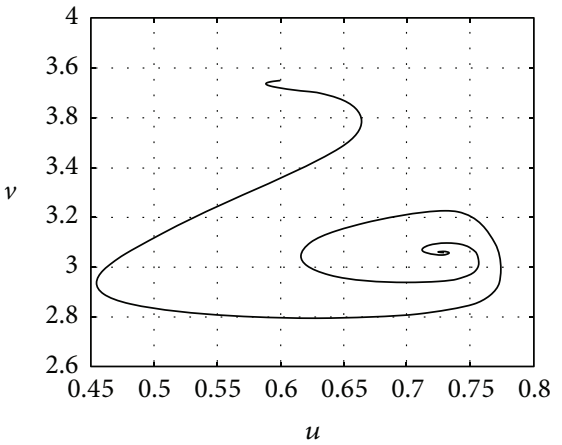

(b3)

Figure 4: Dynamical behavior of model (5). $D_{12}=-0.365$ (the first row); $D_{12}=-0.5$ (the second row); and other parameters are fixed as (27) in the text. (a1), (b1) Two categories of Turing patterns of prey $u$ at $t=10000$; (a2), (b2) two categories of Turing patterns of predator $v$ at $t=10000$; and (a3), (b3) phase portraits.

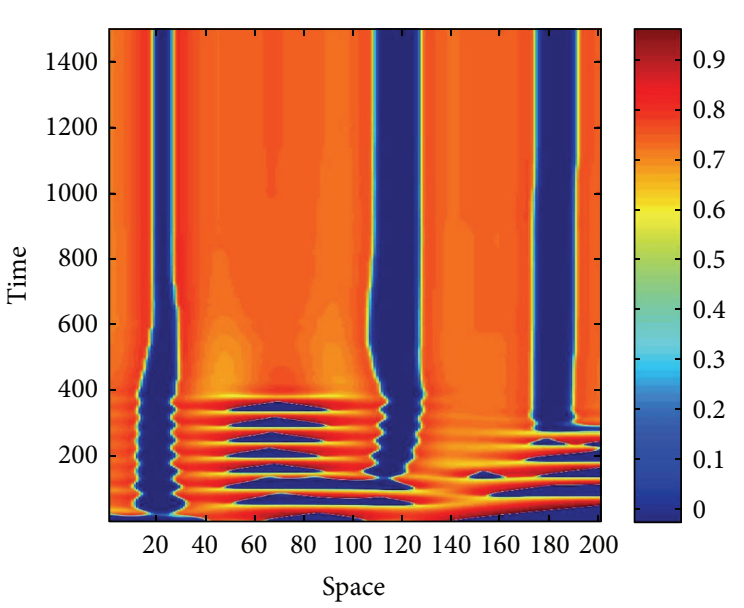

(a)

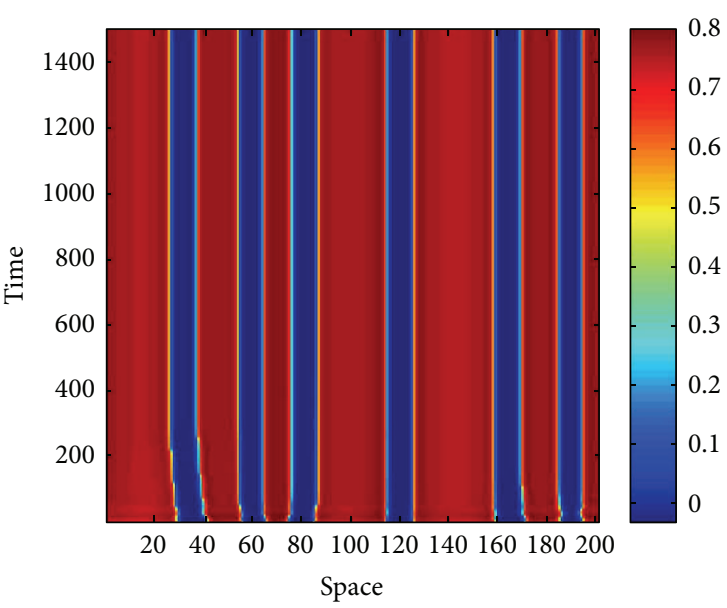

(b)

FIGURE 5: Spacetime plots of prey $u$ of model (5). The time interval shown is 1500 . (a) $D_{12}=-0.365$ and (b) $D_{12}=-0.5$. Other parameters are fixed as (27) in the text.

velocity of the initial random perturbations is thus of the order of $5 \times 10^{-4}$ space units per time unit. Also the system is then integrated for $10^{5}$ time steps, and some images are saved.

From Figure 6(a), we can see that a target wave pattern emerges after perturbation of the stationary solutions $u^{*}$ and $v^{*}$ of model (5). Some iterations later, it breaks towards the core, and a spot pattern (interior) with target wave pattern (exterior) occurs (see Figure 6(b)). Then one can see that after the symmetrical target-spotted mixture patterns (see Figures 6(c) and 6(d)), it grows slightly and the spots increase with time (see Figures 6(e) and 6(f)). From Figure 6, we observe that when the iteration time is large enough, the timeindependent striped-like and target-spotted mixed patterns take a long time to settle down and stay stable. It finally prevails over the whole domain at $t=1000$. 


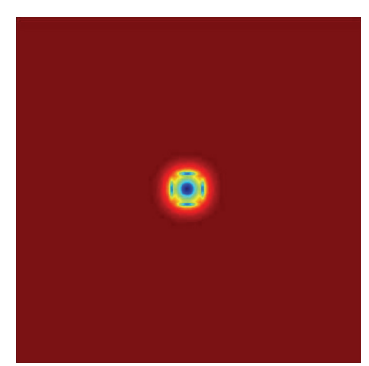

(a)

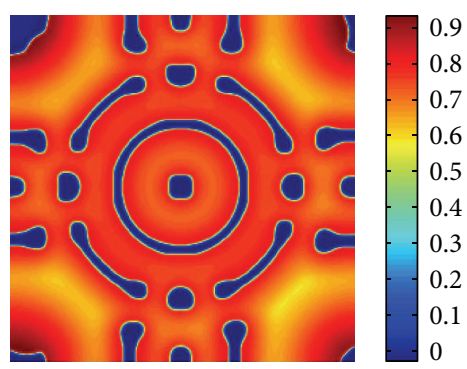

(d)

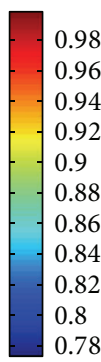

.78

0.9
0.8
0.7
0.6
0.5
0.4
0.3
0.2
0.1
0

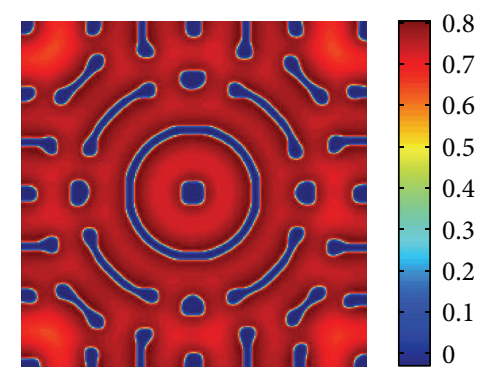

(e)

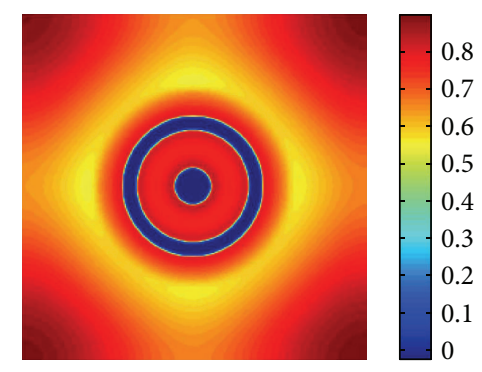

(b)

)

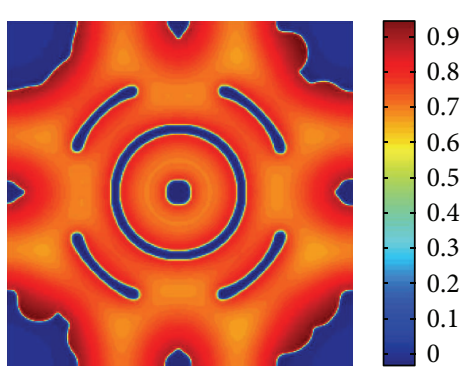

(c)

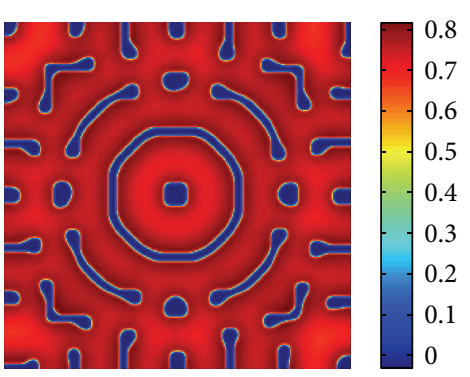

(f)

FIGURE 6: The process of pattern formation of prey $u$ in model (5) with $D_{12}=-0.5$; other parameters are fixed as (27) in the text. Time steps: (a) $t=10$; (b) $t=105$; (c) $t=151$; (d) $t=179$; (e) $t=300$; and (f) $t=1000$.

Figure 6 illustrates the formation of the spatial patterns behavior of prey $u$ in two dimensions with self- and crossdiffusion, where the initial condition (at $t=0$ ) adds random and nonuniform small perturbations to the equilibrium values.

\section{Conclusions and Remarks}

In this paper, we investigate the pattern formation of a Holling-type-II predator-prey interacting model with an Allee effect in prey population and with self- and crossdiffusion under the zero-flux boundary conditions. We show that cross-diffusion in both predator and prey species tends to stabilize the predation model. That is to say, the unstable equilibrium $\left(u^{*}, v^{*}\right)$ of model (5) becomes stable under certain conditions. Moreover, the numerical results show that model (5) dynamics exhibits a cross-diffusion controlled formation growth not only to spotted-striped, but also to spotted pattern replication.

By a series of analysis and numerical simulations, we find that the evolutionary process depends on several parameters. In this paper, we fix the values of $\alpha, \beta, \gamma, \delta, D_{11}, D_{22}$, and $D_{21}$ as (27) and perform a large number of computer simulations corresponding to different $D_{12}$. The results show that model (5) has rich spatiotemporal patterns and different patterns appear on account of the change of cross-diffusion coefficient $D_{12}$. Biological speaking, cross-diffusion may have effect on the distribution of the species. It is also shown that if the equilibrium state $\left(u^{*}, v^{*}\right)$ of model (5) with no diffusion is unstable, then the corresponding uniform steady state of the model with self- and cross-diffusion can be made stable by changing cross-diffusion coefficients. Therefore, we hope that the results presented here will be useful for studying the dynamical complexity of ecosystems or physical systems.

\section{Acknowledgment}

The author is grateful to the reviewers and the editor of this paper for their helpful comments and suggestions.

\section{References}

[1] J. D. Murray, Mathematical Biology, Springer, New York, NY, USA, 3rd edition, 2003.

[2] A. J. Lotka, Elements of Physical Biology, Williams \& Wilkins, Baltimore, Md, USA, 1925.

[3] V. Volterra, "Fluctuations in the abundance of a species considered mathematically," Nature, vol. 118, no. 2972, pp. 558-560, 1926.

[4] C. S. Holling, "The components of predation as revealed by a study of small-mammal predation of the European pine sawfly," The Canadian Entomologist, vol. 91, no. 5, pp. 293-320, 1959.

[5] C. S. Holling, "Some characteristics of simple types of predation and parasitism," The Canadian Entomologist, vol. 91, no. 7, pp. 385-398, 1959.

[6] M. A. Aziz-Alaoui and M. Daher Okiye, "Boundedness and global stability for a predator-prey model with modified LeslieGower and Holling-type II schemes," Applied Mathematics Letters, vol. 16, no. 7, pp. 1069-1075, 2003.

[7] R. Xu, M. A. J. Chaplain, and F. A. Davidson, "Periodic solutions for a predator-prey model with Holling-type functional response and time delays," Applied Mathematics and Computation, vol. 161, no. 2, pp. 637-654, 2005.

[8] R. Yafia, F. El Adnani, and H. T. Alaoui, "Limit cycle and numerical similations for small and large delays in a predatorprey model with modified Leslie-Gower and Holling-type II 
schemes," Nonlinear Analysis: Real World Applications, vol. 9, no. 5, pp. 2055-2067, 2008.

[9] S. Zhang, D. Tan, and L. Chen, "Chaos in periodically forced Holling type II predator-prey system with impulsive perturbations," Chaos, Solitons \& Fractals, vol. 28, no. 2, pp. 367-376, 2006.

[10] V. Krrivan and J. Eisner, "The effect of the Holling type II functional response on apparent competition," Theoretical Population Biology, vol. 70, no. 4, pp. 421-430, 2006.

[11] W. Ko and K. Ryu, "Qualitative analysis of a predator-prey model with Holling type II functional response incorporating a prey refuge," Journal of Differential Equations, vol. 231, no. 2, pp. 534-550, 2006.

[12] Y. Pei, L. Chen, Q. Zhang, and C. Li, "Extinction and permanence of one-prey multi-predators of Holling type II function response system with impulsive biological control," Journal of Theoretical Biology, vol. 235, no. 4, pp. 495-503, 2005.

[13] P. A. Stephens and W. J. Sutherland, "Consequences of the Allee effect for behaviour, ecology and conservation," Trends in Ecology \& Evolution, vol. 14, no. 10, pp. 401-405, 1999.

[14] P. A. Stephens, W. J. Sutherland, and R. P. Freckleton, "What is the Allee effect?” Oikos, vol. 87, no. 1, pp. 185-190, 1999.

[15] F. Courchamp, T. Clutton-Brock, and B. Grenfell, "Inverse density dependence and the Allee effect," Trends in Ecology \& Evolution, vol. 14, no. 10, pp. 405-410, 1999.

[16] W. C. Allee, Animal Aggregations: A Study in General Sociology, AMS Press, New York, NY, USA, 1978.

[17] B. Dennis, "Allee effects: population growth, critical density, and the chance of extinction," Natural Resource Modeling, vol. 3, no. 4, pp. 481-538, 1989.

[18] M. A. Lewis and P. Kareiva, "Allee Dynamics and the Spread of Invading Organisms," Theoretical Population Biology, vol. 43, no. 2, pp. 141-158, 1993.

[19] M. R. Owen and M. A. Lewis, "How predation can slow, stop or reverse a prey invasion," Bulletin of Mathematical Biology, vol. 63 , no. 4, pp. 655-684, 2001.

[20] M.-H. Wang and M. Kot, "Speeds of invasion in a model with strong or weak Allee effects," Mathematical Biosciences, vol. 171, no. 1, pp. 83-97, 2001.

[21] S. V. Petrovskii, A. Y. Morozov, and E. Venturino, "Allee effect makes possible patchy invasion in a predator-prey system," Ecology Letters, vol. 5, no. 3, pp. 345-352, 2002.

[22] S.-R. Zhou, Y.-F. Liu, and G. Wang, “The stability of predatorprey systems subject to the Allee effects," Theoretical Population Biology, vol. 67, no. 1, pp. 23-31, 2005.

[23] J. Shi and R. Shivaji, "Persistence in reaction diffusion models with weak Allee effect," Journal of Mathematical Biology, vol. 52, no. 6, pp. 807-829, 2006.

[24] A. Morozov, S. Petrovskii, and B.-L. Li, "Spatiotemporal complexity of patchy invasion in a predator-prey system with the Allee effect," Journal of Theoretical Biology, vol. 238, no. 1, pp. 18-35, 2006.

[25] L. Berec, E. Angulo, and F. Courchamp, "Multiple Allee effects and population management," Trends in Ecology \& Evolution, vol. 22, no. 4, pp. 185-191, 2007.

[26] C. Çelik and O. Duman, "Allee effect in a discrete-time predatorprey system," Chaos, Solitons \& Fractals, vol. 40, no. 4, pp. 19561962, 2009.

[27] J. Zu and M. Mimura, "The impact of Allee effect on a predatorprey system with Holling type II functional response," Applied
Mathematics and Computation, vol. 217, no. 7, pp. 3542-3556, 2010.

[28] E. González-Olivares, H. Meneses-Alcay, B. González-Yañez, J. Mena-Lorca, A. Rojas-Palma, and R. Ramos-Jiliberto, "Multiple stability and uniqueness of the limit cycle in a Gause-type predator-prey model considering the Allee effect on prey," Nonlinear Analysis: Real World Applications, vol. 12, no. 6, pp. 2931-2942, 2011.

[29] J. Wang, J. Shi, and J. Wei, "Predator-prey system with strong Allee effect in prey," Journal of Mathematical Biology, vol. 62, no. 3, pp. 291-331, 2011.

[30] J. Wang, J. Shi, and J. Wei, "Dynamics and pattern formation in a diffusive predator-prey system with strong Allee effect in prey," Journal of Differential Equations, vol. 251, no. 4-5, pp. 1276-1304, 2011.

[31] Y. Cai, W. Wang, and J. Wang, "Dynamics of a diffusive predator-prey model with additive Allee effect," International Journal of Biomathematics, vol. 5, no. 2, 11 pages, 2012.

[32] W. M. Wang, Y. L. Cai, Y. N. Zhu, and Z. G. Guo, "Allee-effectinduced instability in a reactiondiffusion predator-prey model," Abstract and Applied Analysis, vol. 2013, Article ID 487810, 10 pages, 2013.

[33] Y. Cai, W. Liu, Y. Wang, and W. Wang, "Complex dynamics of a diffusive epidemic model with strong Allee effect," Nonlinear Analysis: Real World Applications, vol. 14, no. 4, pp. 1907-1920, 2013.

[34] J. B. Shukla and S. Verma, "Effects of convective and dispersive interactions on the stability of two species," Bulletin of Mathematical Biology, vol. 43, no. 5, pp. 593-610, 1981.

[35] V. N. Biktashev, J. Brindley, A. V. Holden, and M. A. Tsyganov, "Pursuit-evasion predator-prey waves in two spatial dimensions," Chaos, vol. 14, no. 4, pp. 988-994, 2004.

[36] E. H. Kerner, "Further considerations on the statistical mechanics of biological associations," Bulletin of Mathematical Biology, vol. 21, pp. 217-255, 1959.

[37] N. Shigesada, K. Kawasaki, and E. Teramoto, "Spatial segregation of interacting species," Journal of Theoretical Biology, vol. 79, no. 1, pp. 83-99, 1979.

[38] J. Chattopadhyay, A. K. Sarkar, and P. K. Tapaswi, "Effect of cross-diffusion on a diffusive prey-predator system-a nonlinear analysis," Journal of Biological Systems, vol. 4, no. 2, pp. 159169, 1996.

[39] B. Dubey, B. Das, and J. Hussain, "A predator-prey interaction model with self and cross-diffusion," Ecological Modelling, vol. 141, no. 1-3, pp. 67-76, 2001.

[40] J. Jorne, "The diffusive Lotka Volterra oscillating system," Journal of Theoretical Biology, vol. 65, no. 1, pp. 133-139, 1977.

[41] Y. Lou and W.-M. Ni, "Diffusion, self-diffusion and crossdiffusion," Journal of Differential Equations, vol. 131, no. 1, pp. 79-131, 1996.

[42] Y. Lou and W.-M. Ni, "Diffusion vs cross-diffusion: an elliptic approach," Journal of Differential Equations, vol. 154, no. 1, pp. 157-190, 1999.

[43] K. Kuto, "Stability of steady-state solutions to a prey-predator system with cross-diffusion," Journal of Differential Equations, vol. 197, no. 2, pp. 293-314, 2004.

[44] K. Kuto and Y. Yamada, "Multiple coexistence states for a preypredator system with cross-diffusion," Journal of Differential Equations, vol. 197, no. 2, pp. 315-348, 2004.

[45] G.-Q. Sun, Z. Jin, Q.-X. Liu, and L. Li, "Pattern formation induced by cross-diffusion in a predator-prey system," Chinese Physics B, vol. 17, no. 11, pp. 3936-3941, 2008. 
[46] G.-Q. Sun, Z. Jin, Y.-G. Zhao, Q.-X. Liu, and L. Li, "Spatial pattern in a predator-prey system with both self- and crossdiffusion," International Journal of Modern Physics C, vol. 20, no. 1, pp. 71-84, 2009.

[47] W. Wang, Y. Lin, L. Zhang, F. Rao, and Y. Tan, "Complex patterns in a predator-prey model with self and cross-diffusion," Communications in Nonlinear Science and Numerical Simulation, vol. 16, no. 4, pp. 2006-2015, 2011.

[48] W. M. Wang, Z. G. Guo, R. K. Upadhyay, and Y. Z. Lin, "Pattern formation in a cross-diffusive Holling-Tanner model," Discrete Dynamics in Nature and Society, vol. 2012, Article ID 828219, 12 pages, 2012.

[49] A. Morozov, S. Petrovskii, and B.-L. Li, "Bifurcations and chaos in a predator-prey system with the Allee effect," Proceedings of the Royal Society B, vol. 271, no. 1546, pp. 1407-1414, 2004.

[50] A. Okubo and S. A. Levin, Diffusion and Ecological Problems: Modern Perspectives, vol. 14 of Interdisciplinary Applied Mathematics, Springer, New York, NY, USA, 2nd edition, 2001.

[51] J. D. Murray, “Discussion: Turing's theory of morphogenesisits influence on modelling biological pattern and form," Bulletin of Mathematical Biology, vol. 52, no. 1-2, pp. 119-152, 1990.

[52] A. Munteanu and R. V. Solé, "Pattern formation in noisy self-replicating spots," International Journal of Bifurcation and Chaos, vol. 16, no. 12, pp. 3679-3685, 2006.

[53] M. R. Garvie, "Finite-difference schemes for reaction-diffusion equations modeling predator-prey interactions in MATLAB," Bulletin of Mathematical Biology, vol. 69, no. 3, pp. 931-956, 2007.

[54] J. E. Pearson, "Complex patterns in a simple system," Science, vol. 261, no. 5118, pp. 189-192, 1993.

[55] S. M. Tobias and E. Knobloch, "Breakup of spiral waves into chemical turbulence," Physical Review Letters, vol. 80, no. 21, pp. 4811-4814, 1998.

[56] M. Bär and M. Or-Guil, "Alternative scenarios of spiral breakup in a reaction-diffusion model with excitable and oscillatory dynamics," Physical Review Letters, vol. 82, no. 6, pp. 1160-1163, 1999.

[57] B. Sandstede and A. Scheel, "Absolute versus convective instability of spiral waves," Physical Review E, vol. 62, no. 6, part A, pp. 7708-7714, 2000.

[58] M. Bär and L. Brusch, "Breakup of spiral waves caused by radial dynamics: eckhaus and finite wavenumber instabilities," New Journal of Physics, vol. 6, pp. 1-23, 2004.

[59] F. Xie, D. Xie, and J. N. Weiss, "Inwardly rotating spiral wave breakup in oscillatory reaction-diffusion media," Physical Review E, vol. 74, no. 2, Article ID 026107, 2006.

[60] F. Rao and W. Wang, "Dynamics of a Michaelis-Menten-type predation model incorporating a prey refuge with noise and external forces," Journal of Statistical Mechanics: Theory and Experiment, vol. 2012, no. 3, Article ID P03014, 2012.

[61] J. A. Sherratt, B. T. Eagan, and M. A. Lewis, "Oscillations and chaos behind predator-prey invasion: mathematical artifact or ecological reality?" Philosophical Transactions of the Royal Society B, vol. 352, no. 1349, pp. 21-38, 1997.

[62] A. B. Medvinsky, S. V. Petrovskii, I. A. Tikhonova, H. Malchow, and B.-L. Li, "Spatiotemporal complexity of plankton and fish dynamics," SIAM Review, vol. 44, no. 3, pp. 311-370, 2002. 


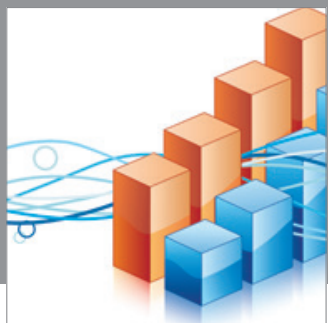

Advances in

Operations Research

mansans

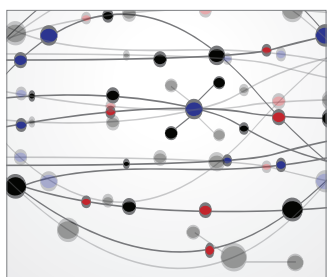

The Scientific World Journal
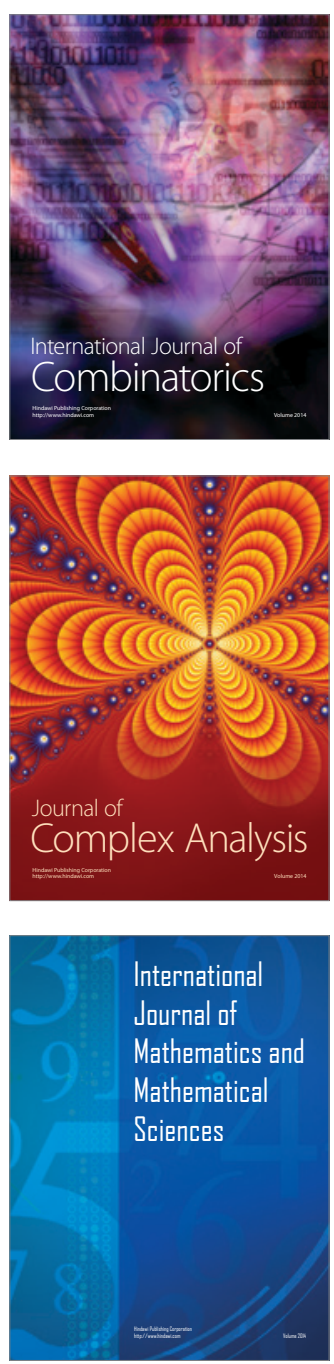
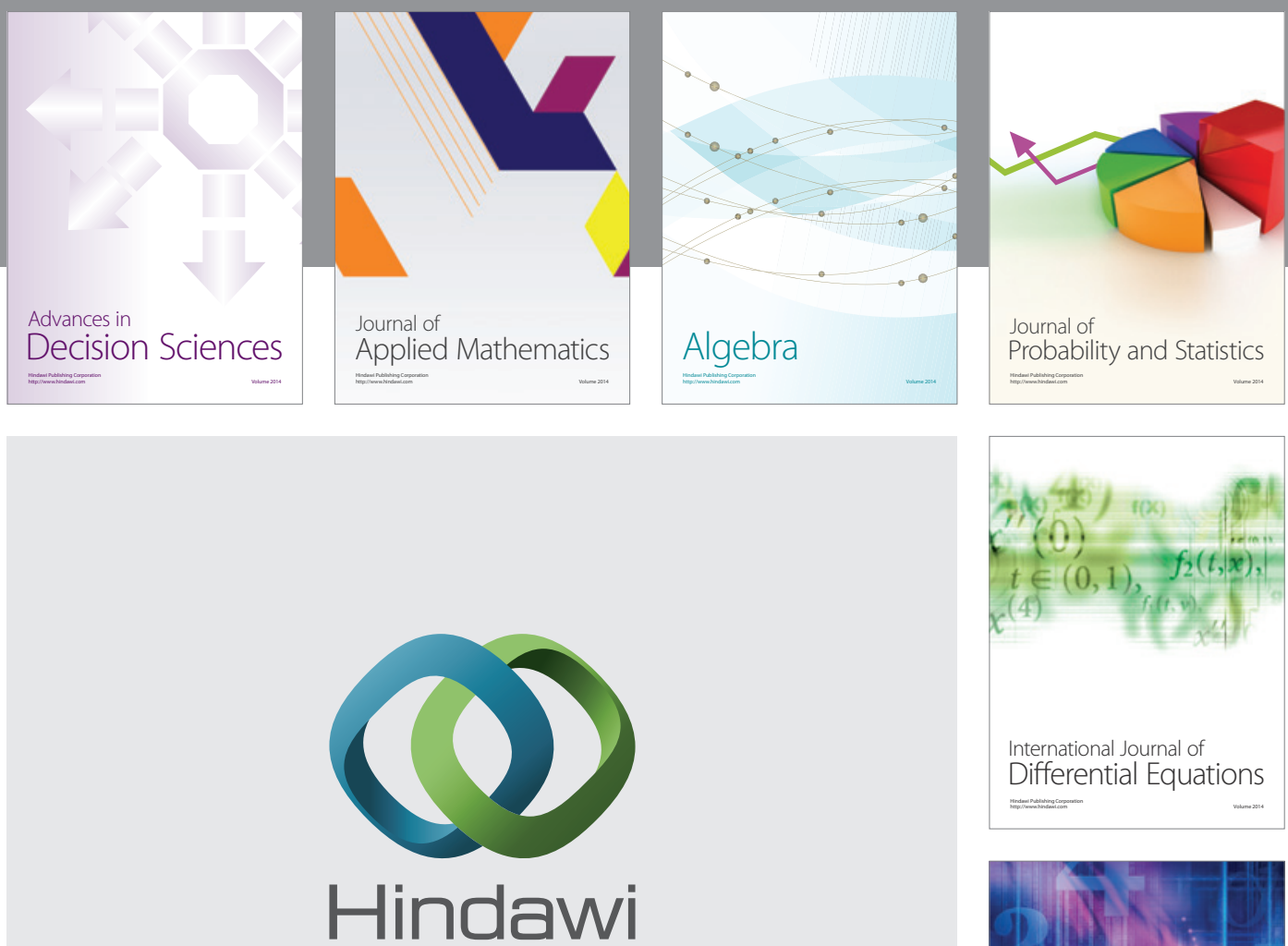

Submit your manuscripts at http://www.hindawi.com
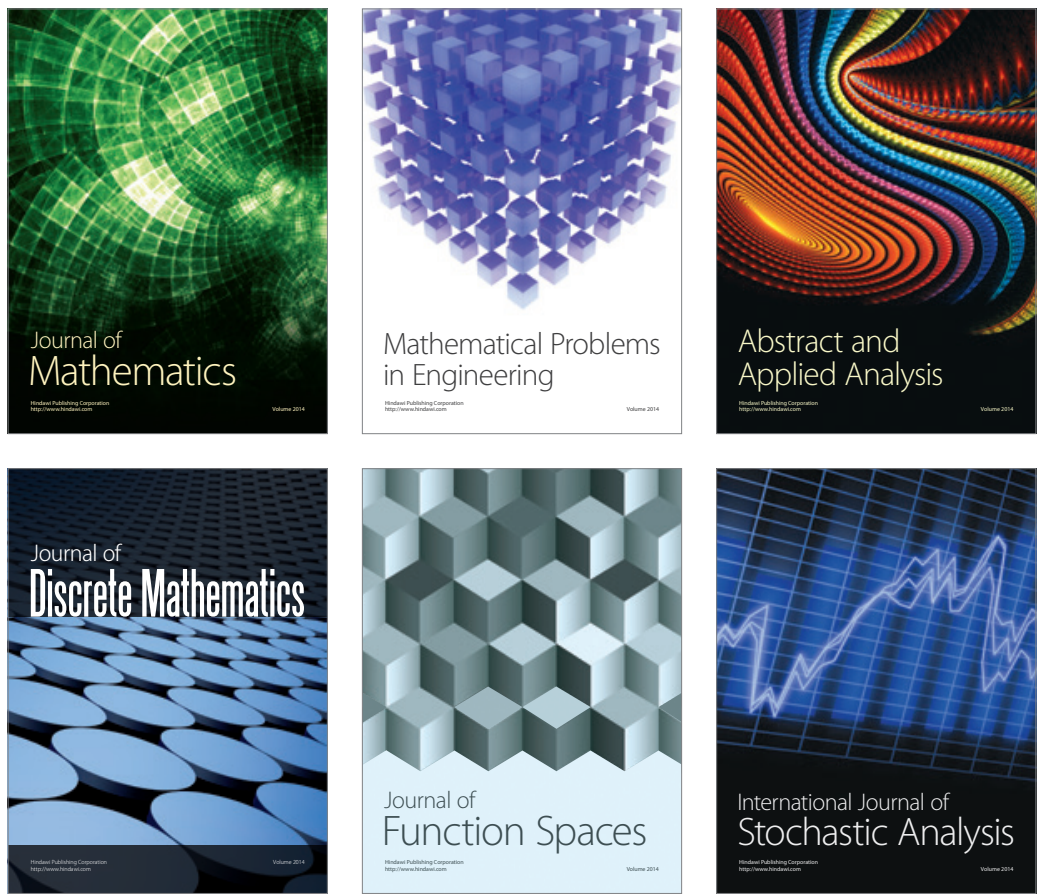

Journal of

Function Spaces

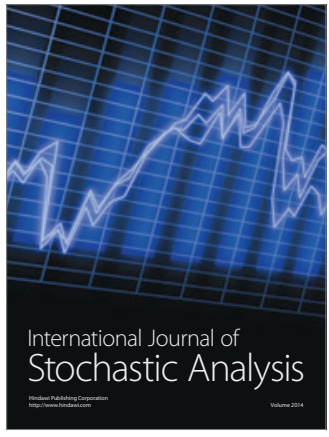

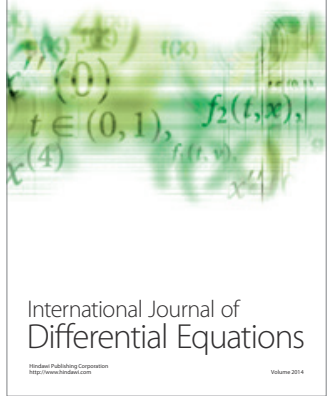
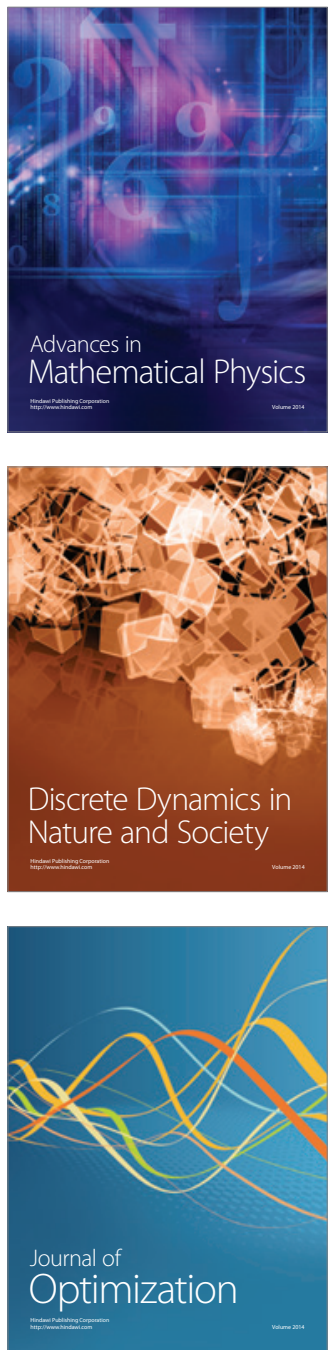